\title{
Association between intestinal and antioxidant barriers in children with cancer
}

\author{
Teresa Stachowicz-Stencel ${ }^{\bowtie}$, Anna Synakiewicz¹, Anna Owczarzak², Aleksandra Sliwinska², \\ Wieslawa Lysiak-Szydlowska² and Anna Balcerska
}

'Department of Paediatrics, Haematology, Oncology and Endocrinology, ${ }^{2}$ Department of Clinical Nutrition, Medical University of Gdansk, Gdańsk, Poland

\begin{abstract}
Objective: Reactive oxygen species (ROS) play a role in cancerogenesis processing and damage tissues. Furthermore, oncological treatment may impair proper function of the gut barrier. The aim of this study was to measure intestinal permeability in children in clinical remission for solid tumours and to search for a possible relationship between free radicals and the intestinal barrier. No such investigation in children has been reported so far. Research Methods and Procedures: The prospective study consisted of 19 paediatric patients with cancer after completion of chemotherapy. 32 healthy children from the outpatients clinics were recruited for measurement of intestinal permeability and antioxidant barrier as a control group. Intestinal permeability was assessed by measurement of urinary lactulose and mannitol after oral challenge. Antioxidant enzymes: superoxide dismutase (SOD) and glutathione peroxidase (GSH-Px) in erythrocytes were assessed. Ischemia modified albumin (IMA) concentration was measured in serum. Results: Cancer patients excreted less mannitol and more lactulose versus controls. The ratio of lactulose to mannitol was significantly higher in oncological children vs control (mean 0.188 and 0.0453 , respectively, $p=0.0006$,). Significantly higher IMA level in the oncological group vs control was noted (mean 123.8 and $87.3 \mathrm{U} / \mathrm{ml}$, respectively, $p=0.0037)$. No correlation between intestinal permeability and oxidative stress barrier was found. Conclusions: Our data shows that intestinal barrier is damaged in paediatric cancer patients after chemotherapy. IMA is believed to play a protective role in the defence against tissue damage. No correlation was found between these two barriers.
\end{abstract}

Key words: intestinal permeability, oxidative stress, childhood cancer Received: 29 July, 2011; revised: 12 February, 2012; accepted: 26 April, 2012; available on-line: 11 May, 2012

\section{INTRODUCTION}

The side-effects of chemotherapy in paediatric oncological patients have been of increasing interest in recent years. More intensive multi-drug chemotherapy may lead to changes in normal tissues, especially those with a high proliferative rate like epithelia of the gastrointestinal tract (Trier, 1968). One of the most uphill post-chemotherapy complications is mucosal barrier injury (MBI), including gastrointestinal mucositis (Vliet et al., 2009; Sonis et al., 2004). It is important to note, however, that this kind of inflammation occurs mainly in the small intestine.
Currently, a five-stage model for MBI is in use. The first two stages are characterised by local inflammation of the intestine, while the third stage is dominated by the loss of enterocytes and submucosal cells. Next is an ulcerative stage during which bacteria and their products are proposed to translocate. The fifth and final stage is the healing one. It leads to a structurally normal mucosal barrier, but it is not known if the healed barrier is fully functional (Sonis et al., 2004; Sonis, 2004). Lesions of the small bowel mucosa are associated with nutritional problems, leading to malnutrition and the necessity to establish a proper diet. They may also cause a reduction of cytostatics doses, implicating a decrease in the overall survival in cancer patients (Elting et al., 2003). A damaged intestinal barrier may allow passage of toxic substances or antigenic macromolecules or bacteria, which may be the reason of some severe adverse-effect such as typhlitis, enterocolitis, infective colitis, perforation, gastrointestinal haemorrhage, ischemic colitis and obstruction (Cronin et al., 2009). However, few reports describe sub-clinical damage and its role in the absorption of nutrients and drugs such as fluoropyrimidines, alkylating compounds, anthracyclines and a taxane/platinum combination (Melichar et al., 2005; Melichar et al., 2007).

Cytostatics may provoke intestinal damage by a decrease in the functioning of the intestinal barrier according to cell proliferation inhibition, and macrophages and neutrophils infiltrate into the inflammatory invasion of endotoxins (Maeda et al., 2010). In a biophylaxis reaction, macrophages and neutrophils infiltrate into the inflammatory area and produce reactive oxygen species (ROS) (Gao et al., 2001; Miyazono et al., 2004). Free radicals, among other effects, play a role in DNA damage and clonogenic cell death in the epithelial layer and in activation of nuclear factor- $x \mathrm{~B}$, which up-regulates inflammatory cytokines such as TNF- $\alpha$, IL-1 $\beta$, IL- 6 and molecules of adhesion. This inflammatory-related transcription factor may be involved in small bowel inflammation (Sonis et al., 2004; Sethi et al., 2008; Mantovani et al., 2008). ROS regulate the paracellular permeability and tight junction opening (Sheth et al., 2003). Defence mechanisms of cells are programmed to protect cells from reactive oxygen species. Superoxide dismutase and glutathione peroxidase belong to the antioxidant repair

e-mail: tsten@gumed.edu.pl

Abbreviations: $\mathrm{BPH}$, benign prostatic hyperplasia; BUN, blood urea nitrogen; GSH-Px, glutathione peroxidase; IMA, ischemia modified albumin; LMER, lactulose-mannitol excretion ratio; MBI, mucosal barrier injury; ROS, reactive oxygen species; SOD, superoxide dismutase. 
system (Gadjeva et al., 2008). This study is a continuation of our earlier study concerning the antioxidant status in children with neuroblastoma and soft tissue sarcomas (Stachowicz-Stencel et al., 2011). One of the most promising oxidative stress markers in paediatric oncological patients seems to be ischemia-modified albumin. Free radicals may modify the $\mathrm{N}$-terminal region of albumin and thus increase IMA levels (Roy et al., 2006). While numerous studies concerning the role of IMA in ischemic disorders have been published, only one paper evaluating the role of this marker in paediatric oncology was found (Stachowicz-Stencel et al., 2011).

The aim of the present study was to measure intestinal permeability in children in clinical remission after chemotherapy for solid tumours. The lactulose-mannitol test is a simple, reliable, non-invasive and safe tool to estimate early-stage intestinal mucosal damage. Thus it seems useful in clinical practice to establish a diet which could help in recovery the proper function of gastrointestinal tract. Furthermore, we made the necessary attempt to find a relationship between antioxidant barriers parameters and the intestinal barrier.

\section{MATERIALS AND METHODS}

Patients. Nineteen paediatric patients with cancer, who were diagnosed and treated in the Department of Paediatrics, Haematology, Oncology and Endocrinology, Medical University of Gdansk, in 2008 and 2009 were enrolled in the study. There were 12 males and 7 females, aged from 2 to 20 years, mean age 10.5 years, median age 9.8 years. All patients had no symptoms of infection (thus excluding any influence of inflammation) at the time of recruiting and before. A complete blood count, C-reactive protein concentration, erythrocyte sedimentation rate, alanine and aspartate transaminases, blood urea nitrogen (BUN), serum creatinine and albumin were performed to check the health status. No patients were receiving vitamin supplementation. The studies were performed at least two years after finishing oncological treatment. This period seems to be sufficient to recover the normal function of damaged organs. All patients received multi-drug chemotherapy. In our study we compared intestinal permeability between healthy control and oncological patients (Table 1).

Control group. Thiry-two healthy children from the outpatient clinics were recruited for measurement of intestinal permeability and antioxidant barrier as a control group (18 girls and 14 boys), aged between 2 and 18 years, mean age 9.97 years, median age 10.92 years. At the time of the study, on the basis of detailed medical history and clinical examination, all children were found to be in complete health with no contemporaneous disease. None of the participants was taking vitamin and/or antioxidant supplements for at least 8 weeks prior to the beginning of the study.

The study was approved by the Ethical Committee of the Medical University of Gdansk.

Laboratory analysis. All assays were performed in the laboratory of the Department of Clinical Nutrition, Medical University of Gdansk, Poland.

Measurement of intestinal permeability. Intestinal permeability was assessed by measurement of urinary lactulose and mannitol after oral challenge. After a 6 hour overnight fast the bladder was emptied completely. Then urinary collection started. Each patient drank a solution containing $10 \mathrm{~g} / \mathrm{l}$ of mannitol and 75 $\mathrm{g} / \mathrm{l}$ of lactulose in quantitles $100 \mathrm{ml} / \mathrm{m}^{2}$ body surface.

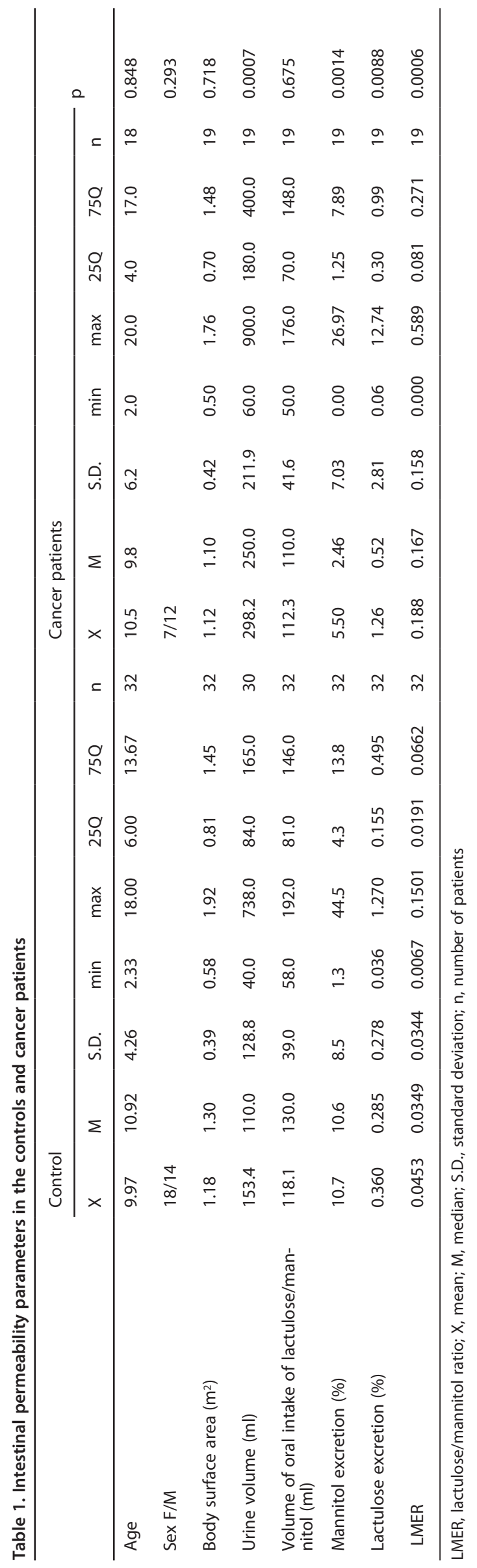


All urine passed for the following $5 \mathrm{~h}$ was collected in urine container. No food was allowed for the duration of the test, although very small amount of water was available. At the end of the urine collection the bladder was emptied completely. The volume of the urine samples was noted and preserved at $-20^{\circ} \mathrm{C}$ until analysed.

Urinary estimations of lactulose and mannitol were measured by the enzymatic analyses described by Northrop et al. (1990) and Lunn et al. (1989), respectively.

The lactulose-mannitol excretion ratio (LMER) was calculated by means of the following ratio after correction for basal samples: LMER=percentage of lactulose excreted in $5 \mathrm{~h} /$ percentage of mannitol excreted in $5 \mathrm{~h}$.

\section{Oxidative stress measurement}

Superoxide dismutase (SOD) assay in erythrocytes. SOD activity in erythrocytes was assessed according to the Ransod (Randox, Crumlin, United Kingdom) procedure. The increase of absorbance was followed at $505 \mathrm{~nm}$ for $3 \mathrm{~min}$ on a UV-VIS spectrophotometer (LKB Pharmacia, Great Britain United Kingdom). Results were expressed in $\mathrm{U} / \mathrm{g} \mathrm{Hb}$.

Glutathione peroxidase (GSHPx) assay in erythrocytes. GSH-PX in erythrocytes was measured according to the Ransel (Randox, Crumlin, United Kingdom) procedure. The decrease of absorbance was recorded at $340 \mathrm{~nm}$ for 3 minutes. GSH-PX was expressed in $\mathrm{U} / \mathrm{g} \mathrm{Hb}$.

Ischemia modified albumin (IMA) in serum. The concentration of ischemia-modified serum albumin was determined by Bhagavan et al. (2003). The absorbance of assay mixtures was read at $470 \mathrm{~nm}$ with a LKB Spectrophotometer. The value of absorbance was given in units $[\mathrm{U} /$ $\mathrm{ml}$ serum] with the use of ACB Roche calibrator of $221 \mathrm{U} / \mathrm{ml}$ as a standard.

Statistical analysis. For each parameter mean $(\mathrm{X})$, median $(\mathrm{M})$, standard deviation (SD), range (min, max), and lower and upper quartile (25Q, $75 \mathrm{Q})$ were calculated. Statistical significance between means for different groups was calculated by one-way analysis of variance (ANOVA), alternatively using the non-parametrical Kruskal-Wallis test, when the variances in groups were not homogeneous (the homogeneity of variance was determinated by the Bartlett's test) or the number of cases in groups was too small to use parametrical tests. Statistical significance between frequencies was calculated by the chi-square test with Yate's correction.
Relation between two parameters was assessed using correlation analysis and Pearson correlation coefficients were calculated. A $p$ value of less than 0.05 was required to reject the null hypothesis. Statistical analysis was performed using EPIINFO Ver. 3.4.3 (08-11-2007) software package.

\section{RESULTS}

We found statistical significant difference for lactulose and mannitol urine excretion between patients and controls $(p=0.0088$ for lactulose and $p=0.0014$ for mannitol). The cancer patients, who received chemotherapy, excreted less mannitol (mean 5.50\%) than the controls (mean 10.7\%). In contrast, excretion of lactulose in the oncological group was higher (mean $1.26 \%$ ) than in the control group (mean $0.360 \%$ ). The ratio of lactulose to mannitol (LMER), which is an estimation of intestinal wall permeability, was significantly higher in children with cancer (mean 0.188) than in the controls (mean 0.0453) $(p=0.0006)$.

In the same group of patients we also measured parameters of oxidative stress barrier. These were: SOD, GSHPx and IMA (Table 2). Our study revealed very interesting results for IMA. The ischemia modified albumin level was significantly higher in the patients with neoplasms (mean $123.8 \mathrm{U} / \mathrm{ml}$ ) than in the control group (mean 87.3 $\mathrm{U} / \mathrm{ml})(p=0.0037)$.

In this particular group of patients there was no correlation between intestinal permeability (considered as LMER) and SOD ( $r=0.17, p=0.510)$, LMER $v$ s GSH-Px $(r=0.12, p=0.629)$ and LMER $v$ IMA $(r=0.09, p=0.723)$.

\section{DISCUSSION}

The measurement of the intestinal barrier integrity seems to be a useful tool in the diagnosing and monitoring of the severity of damage of the intestinal mucosa in neoplastic diseases. We have attempted here to establish of useful markers for the antioxidant barrier in paediatric oncological patients. It is known that during cancer both the intestinal and antioxidant barriers are damaged (Van Huyen et al., 1998; Brown \& Mayer, 2007). For this reason we made an attempt to find a relationship between parameters of these two barriers.

As we examined oncological paediatric patients, who very often are in a poor physical and psychological condition, it was necessary to find a safe and non-invasive method of measurement of small bowel permeability. The 
lactulose-mannitol test meets these criteria and is used in clinical practice (Katz et al., 1989; Zhang et al., 2000). It is assessed by measuring orally administered substances in urine. There are a few hypotheses concerning the passage of substances of different sizes through the mucosa. In healthy people small molecules pass through small and large size pores, while large molecules only via large ones. Another theory describes paracellular route through intercellular junctions (e.g the lactulose) and transcellular route through cellular membrane (e.g the mannitol). The single paracellular permeation model, which is another potential explanation, tells about the tightness of intercellular tight junctions between epithelium cells and an increase of sugar migration from crypts to the villus tip (Brown \& Mayer, 2007; Vilela et al., 2008). It was observed that in children with diarrhoea there was an increase in the mucosal permeability for lactulose and a decrease for mannitol, which resulted in an increase of LMER (Zhang et al., 2003). Pearson et al. (1984) noticed the same situation in paediatric patients with acute lymphoblastic leukemia. Our study is in agreement with those authors. We observed reduced permeation of small molecules such as mannitol and an increase for the larger lactulose. The lactulose to mannitol ratio was significantly higher in cancer patients than in the control group. A study of Pledger et al. (1988) on a group of patients with childhood tumours of different histological types revealed decreased permeation of mannitol, but no changes in the intestinal permeablity of lactulose. The authors made the suggestion that the reason for the observed difference were various types of cytostatics and treatment schedule (continuous or pulses of chemotherapy). In contrast, Benjamin et al. (2008), who examined patients with Crohn's disease, reported no difference in mannitol secretion, but a significant increase in lactulose excretion. For this reason the LMER was also abnormal. All measurements were compared with a control group. Similar findings were observed in studies of Pearson et al. (1982) and Katz et al. (1989). The authors suggest that the lack of difference in mannitol urine excretion between patients with Crohn's disease and healthy controls is connected with the mode of sugar transmission through the intestinal mucosa. Mannitol uses tight junctions in the villous tips, not the pores in the villous crypts as lactulose does. This could be a reason for such result. It is described that, in patients with Crohn's disease, generally there is no villous atrophy, so mannitol excretion may be unaffected in this situation. In oncological patients there is diffuse villous atrophy, so both lactulose and mannitol urine excretion is abnormal (Van Huyen et al., 1998).

It is widely known that the intestinal mucosa in normal conditions is an absorbent and a defense organ. In patients with cancer it is often observed that malnutrition leads to cachexia; the cachexia-anorexia syndrome is associated with lack of ability of using aggressive chemotherapy and leads to poor response to therapy (Argilés, 2005). In malnutrition, the small intestine morphology and physiology change (Monteleone et al., 2004). Some studies show an increased risk of systemic infections and post-operative sepsis in malnourished patients (Megulid et al., 1988; Deitch, 1992).

The intestinal mucosal barrier may be disrupted in increased oxidative stress condition, leading to its increased permeability (Musch et al., 2006). During oxidative stress there is an imbalance between generation of ROS and the antioxidant defence mechanisms (Hamer et al., 2009; Stocker \& Keaney, 2004), as evidenced by various oxidative stress biomarkers such as GSH-Px, SOD and IMA.
SOD is the first line of defence against production of ROS, which eliminates the superoxide radical $\left(\mathrm{O}_{2}^{-}\right)$. GSH-Px and catalase together detoxify $\mathrm{H}_{2} \mathrm{O}_{2}$ by converting it into water and molecular oxygen (Michiels et al., 1994). In the present study SOD activity in erythrocytes of paediatric cancer patients was lower than in the control group. The decreased activity of SOD causes accumulation of cellular $\mathrm{O}_{2}^{-}$, damages mitochondrial membranes and leads to apoptosis of cancer cells (Huang et al., 2000). The similar observations were made by Gadjeva et al. (2008) who described significantly lower erythrocyte SOD activity in patients with melanoma, and Kato et al. (2003) in adult patients with leukaemia. By contrast, in a study by Devi et al. (2000), on children and adults with different types of leukaemias the superoxide dismutase activity was higher versus controls. Badjatia et al. (2009) suggested that lower levels of antioxidants in patients with urothelial carcinoma of the bladder were connected with progression of cancer. Other authors thought that this condition may be related to the scavenging of lipid peroxides and their sequestration by tumour cells (Sharma et al., 2007). In patients with gastric cancer SOD and GSH-Px activities were lower. Superoxide is transmitted through membranes and may have destructive influence on sites far from the tumour. In cancer patients lipid peroxidation of erythrocytes is probably due to $\mathrm{O}_{2}-$ produced by the tumour and low activity of SOD. The low level of GSH-Px may be in relation with increased scavenging of lipid peroxides (Arivazhagan et al., 1997). However, observations made by Dursun et al. (2006) concerning adult patients with oesophagal and gastric cancer showed high activity of SOD but low activity of GSH-Px in erythrocytes. The authors explained that the increased activity of SOD leads to an increase in the conversion of $\mathrm{O}_{2}^{-}$into $\mathrm{H}_{2} \mathrm{O}_{2} . \mathrm{H}_{2} \mathrm{O}_{2}$ may be converted into hypochlorous acid and hydroxyl radical $(\mathrm{OH} \cdot)$ by myeloperoxidase. $\mathrm{OH}$ as a more reactive radical may cause oxidative damage (Dursun et al., 2006).

To the best of our knowledge, there is no published study that describes relation of IMA to intestinal permeability in cancer. Our study revealed significantly increased levels of IMA in cancer patients. Most likely, ischemic tissues modify the albumin $\mathrm{N}$-terminus making it unable to bind cobalt (Bar-Or et al., 2001; Polk et al., 2008). Free radicals may modify the $\mathrm{N}$-terminal region of albumin resulting in increased in IMA levels (Cichota et al., 2008; Roy et al., 2006). We are aware of only one report evaluating IMA in prostatic diseases. The authors observed a slight increase in IMA level in prostatic cancer and a significant increase in benign prostatic hyperplasia $(\mathrm{BPH})$. The cause of the increase IMA may be inflammation connected with $\mathrm{BPH}$ that may lead to release of free radicals (Mastella et al, 2009). It is suggested that very low or very high serum albumin levels may influence IMA concentration and lead to unreliable results (Apple et al., 2005; Gaze et al., 2006). Some authors showed a statistically significant negative correlation between serum albumin and IMA in both healthy adult outpatients and in patients with a suspicion of acute coronary syndrome (Lippi et al., 2007; Lee et al., 2007). In our previous paper, we observed the same inverse relationship between IMA levels and serum albumin concentrations in pediatric cancer patients (Stachowicz-Stencel et al., 2011).

In summary, our data shows that the intestinal barrier is damaged in paediatric cancer patients after chemotherapy. As to the oxidative stress barrier parameters, only IMA level was significantly higher in the oncological patients after treatment vs controls. Further studies 
are needed in order to improve its role as a protective substance against damage of tissue. No correlation was observed between the antioxidant and intestinal barriers.

Author Contributions: All authors confirm that they have contributed to the intellectual content of this paper and have met the following requirements : (a) significant contributions to the conception and design, acquisition of data, or analysis and interpretation of data; (b) drafting or revising the article for intellectual content; and (c) final approval of the published article.

Authors' Disclosures of Potential Conflicts of Interest: None declared.

Role of Sponsor: The funding organizations played no role in the design of the study, choice of enrolled patients, review and interpretation of data, or preparation or approval of manuscript.

\section{REFERENCES}

Apple FS, Wu AH, Mair J, Ravkilde J, Panteghini M, Tate J et al. (2005) Committee on standarization of markers of cardiac damage of the IFCC. Future biomarkers for detection of ischemia and risk stratification in acute coronary syndrome. Clin Chem 51: 810-824.

Argilés JM (2005) Cancer-associated malnutrition. Eur J Oncol Nurs 9: 39-50.

Arivazhagan S, Kavitha K, Nagini S (1997) Erythrocyte lipid peroxidation and antioxidants in gastric cancer patients. Cell Biochem Funct 15: $15-18$.

Badjatia N, Satyam A, Singh P, Seth A, Sharma A (2010) Altered antioxidant sttaus and lipid peroxidation in indian patients with urothelial bladder carcinoma. Urol Oncol 28: 360-367.

Bar-Or D, Curtis G, Rao N, Bampos N, Lau E (2001) Characterization of the $\mathrm{Co}(2+)$ and $\mathrm{Ni}(2+)$ binding amino-acid residues of the $\mathrm{N}$-terminus of human albumin. An insight into the mechanism of a new assay for myocardial ischemia. Eur J Biochem 268: 42-47.

Benjamin J, Makharia GK, Ahuja V, Kalaivani M, Joshi YK (2008) Intestinal permeability and its association with the patient and disease characteristics in Crohn's disease. World J Gastroenterol 14: 13991405.

Bhagavan NV, Lai EM, Rios PA, Yang J, Ortega-Lopez AM, Shinoda $\mathrm{H}$ et al. (2003) Evaluation of human serum albumin cobalt binding assay for the assessment of myocardial ischaemia and myocardial infarction. Clin Chem 49: 581-585.

Brown SJ, Mayer L (2007) The immune response in inflammatory bowel disease. Am J Gastroenterol 102: 2058-2069.

Cichota LC, Moresco RN, Duarte MMMF, Paz da Silva JE (2008) Evaluation of ischemia-modified-albumin in anemia associated to chronic kidney disease. J Clin Lab Anal 22: 1-5.

Cronin CG, O'Connor M, Lohan DG, Kaene M, Roche C, Bruzzi JF et al. (2009) Imaging of the gastrointestinal complications of systemic chemotherapy. Clin Radiol 64: 724-733.

Deitch EA (1992) Multiple organ failure. Pathophysiology and potential future therapy. Ann Surg 216: 117-134.

Devi GS, Prasad MH, Saraswathi I, Raghu D, Rao DN, Reddy PP (2000) Free radicals antioxidant enzymes and lipid peroxidation in different types of leukemias. Clin Chim Acta 293: 53-62.

Dursun H, Bilici M, Uyanik A, Okcu N, Akyuz M (2006) Antioxidant enzyme activities and lipid peroxidation levels in erythrocytes of patients with oesophageal and gastric cancer. I Int Med Res 34: 193-199.

Elting LS, Cooksley C, Chambers M, Cantor SB, Manzullo E, Rubenstein EB (2003) The burdens of cancer therapy. Clinical and economic outcomes of chemotherapy-induced mucositis. Cancer 98: 1531-1539.

Gadjeva V, Dimov A, Georgieva N (2008) Influence of therapy on the anioxidant status in patients with melanoma. J Clin Pharm Therap 33: 179-185.

Gao F, Ueda S, Horie T (2001) Effect of synthetic analog of prostaglandin E1 on the intestinal mucosa of methotrexate-treated rats. Anticancer Res 21: 1913-1917.

Gaze DC, Crompton L, Collinson P (2006) Ischemia-modified albumin concentrations should be interpreted with caution in patients with low serum albumin concentrations. Med Princ Pract 15: 322-324.

Hamer HM, Jonkers DMAE, Bast A, Vanhoutivin SALW, Fischer MAJG, Kodde A et al. (2009) Butyrate modulates oxidative stress in the colonic mucosa of healthy humans. Clin Nutrition 28: 88-93.

Huang P, Feng L, Oldham EA, Keating MJ, Plunkett W (2000) Superoxide dismutase as a target for the selective killing of the cancer cells. Nature 407: 390-395.

Kato M, Minakami H, Kuroiwa M, Kobayashi Y, Oshima S, Kozawa $\mathrm{K}$ et al. (2003) Superoxide radical generation and $\mathrm{Mn}$ - and $\mathrm{Cu}-\mathrm{Zn}$ superoxide dismutases activities in human leukemic cells. Hematol Oncol 21: 11-16.

Katz KD, Hollander D, Vadheim CM, McElree C, Delahunty T, Dadufalza VD et al. (1989) Intestinal permeability in patients with Crohn's disease and their healthy relatives. Gastroenterology 97: 927931.

Lee YW, Kim HJ, Cho YH, Shin HB, Choi TY, Lee YK (2007) Application of albumin-adjusted ischemia modified albumin index as an early screening marker for acute coronary syndrome. Clin Chim Acta 384: 24-27.

Lippi G, Montagnana M, Salvagno GL, Guidi GC (2007) Standardization of ischemia-modified albumin testing: Adjustment for serum albumin. Clin Chem Lab Med 45: 261-262.

Lunn PG, Northrop CA, Northrop AJ (1989) Automated enzymatic assays for the determination of intestinal permeability probes in urine. 2. Mannitol . Clin Chim Acta 183: 163-170.

Maeda T, Miyazono Y, Ito K, Hamada K, Sekine S, Horie T (2010) Oxidative stress and enhanced paracellular permeability in the small intestine of methotrexate-treated rats. Cancer Chemother Pharmacol 65: 1117-1123.

Mantovani A, Allavena P, Sica A, Balkwill F (2008) Cancer-related inflammation. Nature 454: 436-444.

Mastella AK, Moresco RN, Bisognin da Silva D, Becker AM, Duarte MM, Giovelli LL, et al (2009) Evaluation of ischemia-modified albumin in myocardial infarction and prostatic diseases. Biomed Pharmacother 63: 762-766.

Meguild MM, Debonis D, Meguld V (1988) Complications of abdominal operations for malignant disease. Am J Surg 156: 341-345.

Melichar B, Dvorak J, Hyšpler R, Zadak Z (2005) Intestinal permeability in the assessment of intestinal toxicity of cytotoxic agents. Chemotherapy 51: 336-338.

Melichar B, Hyšpler R, Dragounova E, Dvorak J, Kalabova H, Ticha A (2007) Gastrointestinal permeability in ovarian cancer and breast cancer patients treated with paclitaxel and platinum. BMC Cancer 7: 55.

Michiels C, Raes M, Toussaint O, Remacle J (1994) Importance of Seglutathione peroxidase, catalase, and $\mathrm{Cu} / \mathrm{Zn}-\mathrm{SOD}$ for cell survival against oxidative stress. Free Radic Biol Med 17: 235-248.

Miyazono Y, Gao F, Horie T (2004) Oxidative stress contributes to methotrexate-induced small intestinal toxicity in rats. Scand J Gastroenterol 39: 1119-1127.

Monteleone P, Carratu R, Carteni M, Generoso M, Lamberti M, De Magistris L et al. (2004) Intestinal permeability is decreased in anorexia nervosa. Molec Psych 9: 76-80.

Musch MW, Walsh-Reitz MM, Chang EB (2006) Roles of ZO-1, occludin, andactin in oxidant-induced barrier disruption. Am J Physiol Gastrointest Liver Physiol 290: G222-G231.

Northrop CA, Lunn PG, Behrens RH (1990) Automated enzymatic assays for the determination of intestinal permeability probes in urine. 1. Lactulose and lactose. Clin Chim Acta 187: 79-88.

Pearson AD, Craft AW, Pledger JV, Eastham EJ, Laker MF, Pearson GL (1984) Small bowel function in acute lymphoblastic leukaemia. Arch Dis Child 59: 460-465.

Pearson AD, Eastham EJ, Laker MF, Craft AW, Nelson R (1982) Intestinal permeability in children with Crohn's disease and coeliac disease. Br Med J (Clin Res Ed) 285: 20-21.

Pledger JV, Pearson AD, Craft AW, Laker MF, Eastham EJ (1988) Intestinal permeability during chemotherapy for childhood tumours. Eur I Pediatr 147: 123-127.

Polk JD, Rael LT, Craun ML, Mains CW, Davis-Merritt D, Bar-Or D (2008) Clinical utility of the cobalt-albumin binding assay in the diagnosis of intestinal ischemia. J Trauma 64: 42-45.

Roy D, Quiles J, Gaze DC, Collinson E, Kaski JC, Baxter GF (2006) Role of reactive oxygen species on the formation of the novel diagnostic marker ischemia modified albumin. Heart 92: 113-114.

Roy D, Quiles J, Gaze DC, Collinson P, Kaski JC, Baxter GF (2006) Role of reactive oxygen species on the formation of the novel diagnostic marker ischemia modified albumin. Heart 92: 113-114.

Sethi G, Sung B, Aggarwal BB (2008) Nuclear factor-kappaB activation, from bench to bedside. Exp Biol Med 233: 21-31.

Sharma A, Ryaiiapa M, Saxena A, Sharma M (2007) Antioxidant status in advanced cervical cancer patients undergoing neoadjuvant chemoradiation. Br J Biomed Sci 64: 23-27.

Sheth P, Basuroy S, Li C, Naren AP, Rao RK (2003) Role of phosphatidylinositol 3-kinase in oxidative stress-induced disruption of tight junctions. J Biol Chem 278: 49239-49245.

Sonis ST (2004) The pathobiology of mucositis. Nat Rev Cancer 4: 277284.

Sonis ST, Elting LS, Keefe D, Peterson DE, Schubert M, Hauer-Jensen M et al. (2004) Perspectives on cancer therapy-induced mucosal injury, pathogenesis measurement, epidemiology, and consequences for patient. Cancer 100: 1995-2025.

Stachowicz-Stencel T, Synakiewicz A, Owczarzak A, AleksandrowiczWrona E, Sliwinska A, Lysiak-Szydlowska W, Balcerska A (2011) The antioxidant status and response to therapy in children with soft tissue sarcomas and neuroblastoma. Pediatr Blood Cancer 57: 561-568. 
Stachowicz-Stencel T, Synakiewicz A, Owczarzak A, Sliwińska A, Aleksandrowicz-Wrona E, Lysiak-Szydowska W et al. (2011) Ischemiamodified albumin as a biochemical marker in children with neuroblastoma and soft tissue sarcomas. J Clin Lab Anal 25: 255-258.

Stocker R, Keaney Jr JF (2004) Role of oxidative modifications in atherosclerosis. Physiol Rev 84: 1381-1478.

Trier JS (1968) Morphology of the epithelium of the small intestine. In: Handbook of Physiology, vol 3, section 6. Washington, DC, American Physiological Society.

Van Huyen JP, Bloch F, Attar A, Levoir D, Kreft C, Molina T et al. (1998) Diffuse mucosal damage in the large intestine associated with Irinotecan (CPT-11) Dig Dis Sci 43: 2649-2651.
Vilela EG, Torres HO, Ferrari ML, Lima AS, Cunha AS (2008) Gut permeability to lactulose and mannitol differs in treated Crohn's disease and celiac disease patients and healthy subjects. BrazJ Med Biol Res 41: 1105-1109.

Vliet MJ, Tissing WJ, Rings EH, Stellaard F, Kamps WA et al. (2009) Citrulline as a marker for chemotherapy induced mucosal barrier injury in pediatric patients. Pediatr Blood Cancer 53: 1188-1194.

Zhang Y, Lee B, Thompson M, Glass R, Cama R I, Figueroa D et al. (2000) Lactulose-mannitol intestinal permeability test in children with diarrhea caused by rotavirus and cryptosporidium. Diarrhea working group, Peru. J Pediatr Gastroenterol Nutr 31: 16-21. 